\title{
Full-waveform inversion with subsurface fractal information and variable model uncertainties
}

\author{
Pedro Carvalho (Federal University of Rio Grande do Norte)*, Gilberto Corso (Federal University of Rio Grande do Norte), \\ Sérgio Luiz (Federal University of Rio Grande do Norte), João de Araújo (Federal University of Rio Grande do Norte) and \\ Liacir Lucena (Federal University of Rio Grande do Norte)
}

Copyright 2019, SBGf - Sociedade Brasileira de Geofísica.

This paper was prepared for presentation at the $16^{\text {th }}$ International Congress of the Brazilian Geophysical Society, held in Rio de Janeiro, Brazil, 19-22 August, 2019.

Contents of this paper were reviewed by the Technical Committee of the $16^{\text {th }}$ International Congress of the Brazilian Geophysical Society and do not necessarily represent any position of the SBGf, its officers or members. Electronic reproduction or storage of any part of this paper for commercial purposes without the written consent of the Brazilian Geophysical Society is prohibited.

\section{Abstract}

Full-waveform inversion (FWI) is a powerful technique with the ability to provide accurate images of the subsurface, however, because it is an ill-posed inverse problem it still faces some difficulties. An FWI approach with great potential is the Bayesian formulation because it has features that are able to mitigate the ill-posedness. Namely, this approach enables the inclusion of a priori knowledge, regularisation and constraints into the FWI. However, for reasons of computational cost and difficulties related to the definition and inclusion of a priori information, this approach has not been widely applied in the literature. In this work, in an attempt to overcome some of these difficulties, it is proposed to vary the prior model and the corresponding uncertainties throughout the inversion coupled with the inclusion of a priori knowledge regarding correlations between the P-velocities (characterised by fractional Brownian motions). In addition to reducing the initial difficulties in defining the uncertainties, our proposal seeks to assist the FWI in overcoming problems at the beginning of the inversion (e.g. caused by the inaccuracy of the initial model) giving initially a greater weight to the a priori information and after that, after these difficulties surpassed, prioritises the information from the observed data. We demonstrate how powerful our proposal is compared to the traditional ones in an adverse situation, in which the data are devoid of low frequencies (below 3 $\mathrm{Hz}$ ) and have a high noise level.

\section{Introduction}

Full-waveform inversion (FWI) is a technique that has been recognised as powerful because its potential to provide more accurate and more detailed images of the subsurface than traditional seismic imaging techniques. Originally proposed by Lailly (1983) and Tarantola (1984), this technique comprises an optimisation problem in which one aims to find the subsurface physical properties that lead to the best fit between modelled and recorded seismograms. Because FWI involves the modelling of the wavefields from the full-wave equation and aims to achieve modelled seismograms as similar as possible to the recorded seismograms, it has a particular ability to explore almost all the information contained in the observed seismograms and consequently to obtain more accurate images of the subsurface. However, because it is an ill-posed inverse problem, nonlinear and with a large number of variables, FWI still faces several obstacles. Although many approaches and strategies have been proposed in the literature to overcome these obstacles (e.g., objective functions different from the traditional the $l_{2}$-norm of residuals, hierarchical multiscale strategies and regularization techniques) some obstacles continue to persist (Virieux and Operto, 2009; Tejero et al., 2015; Li and Demanet, 2016; Virieux et al., 2017).

One approach with great potential is, for instance, the Bayesian formulation of the FWI (Tarantola, 1987; Mosegaard and Tarantola, 2002). This approach has several features that are crucial in mitigating the illposedness. Namely, this approach enables the inclusion of a priori knowledge, regularisation and constraints. Particularly noteworthy are the addition of a priori information and regularisation which have been referred in the literature as particularly important in mitigating some problems such as cycle-skipping, and problems caused by high noise levels in the observed data. However, because it is impracticable to apply this approach genuinely in FWI due to its high computational cost and because difficulties in defining and incorporating a priori knowledge, this approach has not been widely used in the literature (Bui-Thanh et al., 2013; Virieux et al., 2017).

In a previous work, (Carvalho et al., 2018), we have found that is exceptionally beneficial to incorporate in the FWI information regarding existing statistical correlations between the $\mathrm{P}$-velocities characterised by fractional Brownian motions (fBm) (Sahimi and Tajer, 2005) in FWI, however, the initial definition of appropriate uncertainties has prevailed a problem. In order to overcome this difficulty, we propose in this work to vary the uncertainties concerning to the model during the inversion process. We propose to begin the inversion with the smallest uncertainties and then gradually to increase them whenever the objective function begins to approaching a possible local minimum (i.e., when the objective function begins to take very close values).

In this way, we are considerably reducing the initial difficulties in defining the model uncertainties (with the attribution of the lowest uncertainties) and, at the same time, we are helping the FWI to overcome the initial difficulties (e.g. caused by the inaccuracy of the initial model) with greater importance (weight) given to the a priori information at the beginning of the inversion. And after these difficulties have been overcome, in line with the Asnaashari et al. (2013) strategy, priority is given to 
information from the observed data (with the reduction of the weight of a priori information).

\section{Methodology}

FWI is a technique formulated as an optimisation problem, in which the aim is to find the subsurface physical properties (e.g., P-velocities in the acoustic approximation) that minimise the differences between modelled and observed data (Lailly, 1983; Tarantola, 1984). In the Bayesian formulation one aims to minimise the following objective function (Tarantola, 1987; Mosegaard and Tarantola, 2002):

$$
\begin{array}{r}
S(m)=\frac{1}{2} \sum_{s=1}^{n s} \sum_{r=1}^{n r}\left(d_{r, s}(m)-d_{r, s}^{o b s}\right)^{\top} C_{D}^{-1}\left(d_{r, s}(m)-d_{r, s}^{o b s}\right) \\
+\frac{1}{2}\left(m-m_{\text {prior }}\right)^{\top} C_{M}^{-1}\left(m-m_{\text {prior }}\right)
\end{array}
$$

where $d_{r, s}(m)$ and $d_{r, s}^{o b s}$ are the modelled data (obtained from the model $m$ ) and the observed seismic data, respectively (recorded at receiver $r$ generated by the source $s$ ), $C_{D}$ and $C_{M}$ are the covariance matrices corresponding to the data and the model respectively, $m_{\text {prior }}$ is the model with a priori information about the model parameters and $n s$ and $n r$ are the number of sources and of receivers, respectively.

Note that the most commonly applied objective function in the literature $\left(l_{2}\right.$-norm of the differences between modelled and observed data) comprises considering only the first term and assuming the covariance matrix $C_{D}$ equal to the identity.

Contrary to what is commonly considered in literature, in this work we propose to vary the uncertainties regarding to the model and the a priori model throughout the optimisation process and, in addition, to incorporate a priori knowledge that in previous works we have found to be valuable information in $\mathrm{FWI}$, more specifically knowledge regarding existing statistical correlations between the $\mathrm{P}$ velocities in depth (in the vertical direction) characterised by fBm (Sahimi and Tajer, 2005; Carvalho et al., 2018).

Our proposal is to start the inversion with small uncertainties and gradually to increase them in depth throughout the optimisation process whenever the objective function tends to converge to possible local minima. We assume the uncertainties to be linearly increasing in depth, but remain small and constant at the surface. We assume that the objective function tends to converge to a local minimum whenever the objective function decreases very little in several consecutive iterations.

Through our proposal, at the beginning of the inversion, the P-velocities are forced not to depart too far from the most probable ones (i.e., from the a priori model) (with the assignment of small uncertainties) and, at the same time, a greater importance is given to the a priori information (given that smaller uncertainties correspond to higher weights for the term of the objective function corresponding to the a priori information: second term of the objective function (1)). In addition, whenever the objective function tends to approach a possible local minimum, we propose to replace the a priori model with the last iteration model and increase the uncertainties. In this way, it contributes to helping the FWI to overcome the initial problems it usually faces, such as cycle-skipping situations caused by the inaccuracy of the initial model. And, once these initial problems have been overcome, a greater importance (weight) is given to the information from the observed data by reducing the weight of the a priori information and simultaneously giving greater freedom to the P-velocities to take quantities further away from the a priori model.

Furthermore, we include the a priori knowledge that the $\mathrm{P}$-velocities follow $\mathrm{fBm}$ in depth (in the vertical direction). This a priori knowledge is included through the covariance matrix corresponding to the model $\left(C_{M}\right)$ based on the following covariance function (2), which represents the covariance between the $\mathrm{P}$-velocities at $t$ and $s$ depths in a given vertical $\mathrm{P}$-velocity profile, and based on the P-velocity uncertainties assumed in the usual Bayesian formulation context (Carvalho et al., 2018):

$$
\operatorname{Cov}\left(B_{t}^{H} B_{s}^{H}\right)=\frac{1}{2}\left(\sigma_{t}^{2}+\sigma_{s}^{2}-\left|\left(\sigma_{t}^{2}\right)^{1 / 2 H}-\left(\sigma_{s}^{2}\right)^{1 / 2 H}\right|^{2 H}\right)
$$

where $B^{H}$ is a continuous Gaussian process which starts at zero and with expectation zero at all positions (which in our case corresponds to a vertical P-velocity profile subtracted from the P-velocity at the surface - initial point), $H \in(0,1)$ is an index - the Hurst index - that characterises the fBm and, $\sigma_{t}^{2}$ and $\sigma_{s}^{2}$ are the a priori variances of the P-velocities at $t$ and $s$ depths respectively, assumed in the usual Bayesian formulation context.

$\mathrm{fBm}$ is a generalisation of a Wiener process or Brownian motion but with increments that may not be independent (Mandelbrot and van Ness, 1968). A fBm is characterised by a parameter, the Hurst index or Hurst exponent $H \in$ $(0,1)$, which describes the degree of correlation between increments and it is governed by the following covariance function:

$$
\operatorname{Cov}\left(B_{t}^{H} B_{s}^{H}\right)=\frac{1}{2}\left(t^{2 H}+s^{2 H}-|t-s|^{2 H}\right)
$$

where, as previously, $B^{H}$ represents a continuous Gaussian process which starts at zero and with expectation zero at all positions, $H \in(0,1)$ is the Hurst index of the $\mathrm{fBm}$ and $t$ and $s$ are the positions (depth).

When the Hurst index is $H<1 / 2$ the increments of the process are negatively correlated whereas when the Hurst index is $H>1 / 2$ the increments are positively correlated. On the other hand, when $H=1 / 2$ the process is a standard Wiener process or Brownian motion.

Thus, if one assumes that at $t$ and $s$ depths the a priori uncertainties (variances) associated with the prior model are $\sigma_{t}^{2}$ and $\sigma_{s}^{2}$, respectively, and assuming that the Pvelocities follow a $\mathrm{fBm}$ in depth, it can be said that: $\operatorname{Cov}\left(B_{t}^{H} B_{t}^{H}\right)=t^{2 H}=\sigma_{t}^{2}$ and $\operatorname{Cov}\left(B_{s}^{H} B_{s}^{H}\right)=s^{2 H}=\sigma_{s}^{2}$. And, therefore, substituting these relationships in equation (3) it can be deduced that the P-velocities at depths $t$ and $s$ are correlated according to the covariance function (2). In agreement with the results found by Sahimi and Tajer (2005) we assume that the subsurface follows a fBm with a Hurst index of about $H=0.35$.

\section{Numerical examples}

In order to demonstrate the potential of our proposal, we present the results of the inversion of a part of the 


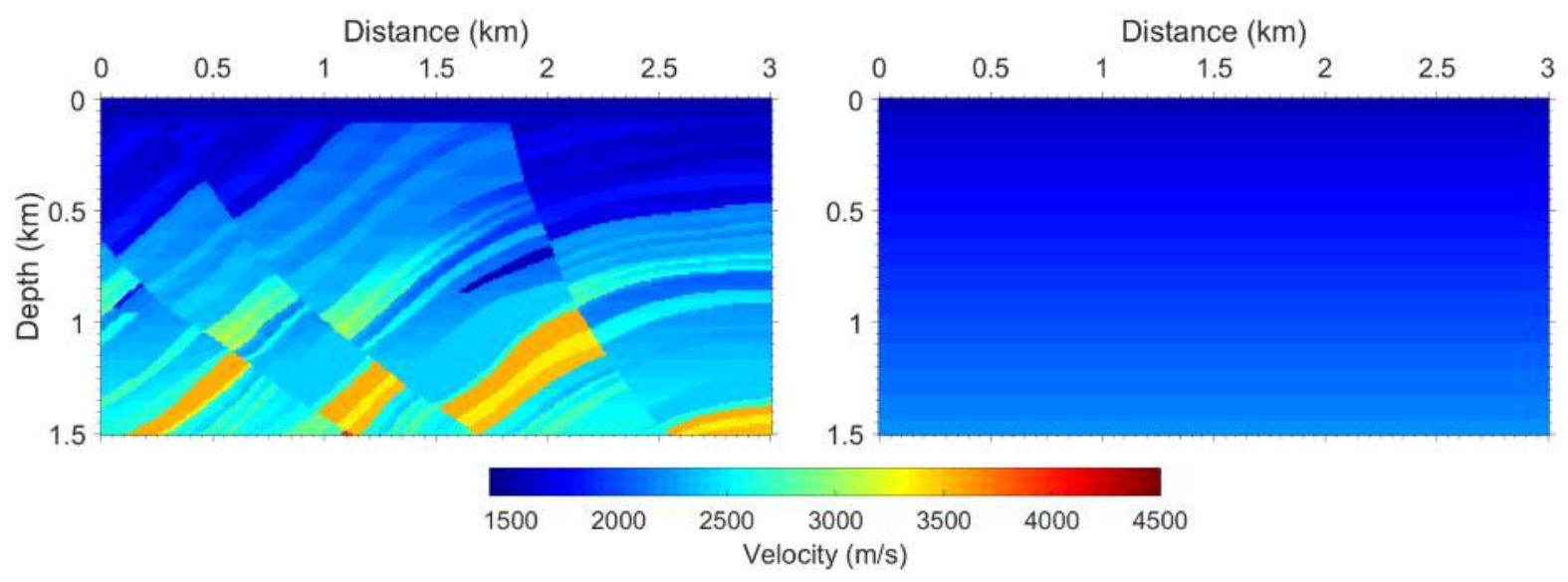

Figure 1 - True P-velocity model (left) and initial P-velocity model (with linearly increasing P-velocities in depth, with $1600 \mathrm{~m} / \mathrm{s}$ at $60 \mathrm{~m}$ and $2200 \mathrm{~m} / \mathrm{s}$ at $1500 \mathrm{~m}$ depth) (right).

Marmousi2 model (Martin et al., 2006) obtained by our methodology and the traditional objective functions.

The true P-velocity model (Figure 1, left) corresponds to a region of $3 \mathrm{~km}$ of extension and $1.5 \mathrm{~km}$ depth and it comprises one of the geologically more complex parts of Marmousi2 consisting mainly of some geological faults. The acquisition geometry consists of 15 sources fired at every $200 \mathrm{~m}$ (between the distances of $100 \mathrm{~m}$ and 2900 $\mathrm{m})$ at $20 \mathrm{~m}$ depth and a total of 144 receivers distributed along the surface $20 \mathrm{~m}$ apart (between the distances of $40 \mathrm{~m}$ and $2960 \mathrm{~m}$ ) at $10 \mathrm{~m}$ depth. The acquisition time was 3.0 seconds. The sources are assumed to be Ricker wavelets with $10 \mathrm{~Hz}$ peak frequency and, for the purpose of simulating a situation close to the real a high-pass filter was applied to remove the frequency components below $3 \mathrm{~Hz}$. The observed seismograms were generated synthetically from the true model and a Gaussian noise was added, resulting observed seismograms with a median signal-tonoise ratio (SNR) of around $12 \mathrm{~dB}$.

A spatial regular discretization of $10 \mathrm{~m}$ was considered. The finite-difference method in the time-domain with fourthorder and second-order accuracy in space and time, respectively, is applied to model the wavefields. In order to simulate an infinite models beyond the edges, the unsplit convolutional perfectly matched layers (C-PML) (Komatitsch and Martin, 2007; Pasalic and McGarry, 2010) absorbing boundary condition is used on all model edges.

In the application of the FWI Bayesian formulation it was assumed that the P-velocities individually follow a normal probability distribution with a mean equal to the prior model and the uncertainties are assumed to be linearly increasing in depth, with a constant standard deviation at the surface of $200 \mathrm{~m} / \mathrm{s}$ (for a confidence level of $99.7 \%$ ). The gradual increase in the uncertainties is achieved by increasing standard deviation increments of $100 / 3 \mathrm{~m} / \mathrm{s}$ at maximum depth up to a maximum of $2000 / 3 \mathrm{~m} / \mathrm{s}$, whenever the objective function tends to converge to local minima. In this work, we assume that the objective function tends to converge to a local minimum whenever in 30 consecutive iterations the objective function decreases less than $0.1 \%$.

Starting from P-velocity model of the Figure 1 (right) as initial model (also assumed as prior model) the P- velocity models presented in Figure 2 were obtained, when the traditional objective function $\left(l_{2}\right.$-norm of the residuals) (Figure 2A), the traditional Bayesian formulation (Figure 2B), Traditional Bayesian formulation with our proposal (Figure $2 \mathrm{C}$ ), Bayesian formulation with a priori knowledge regarding statistical correlations between the Pvelocities (Figure 2D) and Bayesian formulation with a priori knowledge regarding statistical correlations between the Pvelocities and with our proposal (Figure 2E) were applied.

As can be seen, the superiority of our proposal over traditional objective functions is clear. With our proposal it was possible to achieve a much more accurate image of the subsurface (Figure 2E), particularly when compared to the P-velocity models provided by the traditional objective functions: $l_{2}$-norm of the residuals (Figure 2A) and traditional Bayesian formulation (Figure 2B), which were unable to provide practically any relevant information about the subsurface beyond $600 \mathrm{~m}$ depth. On the contrary, from our proposal, it was possible to identify a large part of the geological structures up to a greater depth (Figure 2E).

It is noteworthy that despite the inclusion of the information regarding statistical correlations between the P-velocities in the traditional Bayesian formulation made it possible to obtain a significantly more accurate image of the subsurface (Figure 2D) only together with our proposal it was possible to achieve a sharper image of the subsurface, particularly at a greater depth (Figure 2E).

Note also that while our proposal when applied only with the traditional Bayesian formulation doesn't lead to significantly better results than the traditional Bayesian formulation (comparing Figure 2C with Figure 2B), our proposal becomes particularly powerful when information about the correlations between the P-velocities is included (Figure 2E).

\section{Conclusions}

We have found that our proposal of assigning variable model uncertainties throughout the inversion process in the Bayesian formulation of the FWI coupled with information regarding statistical correlations between the $\mathrm{P}$-velocities characterised by $\mathrm{fBm}$ is a powerful strategy, enabling to achieve quite more accurate images of the subsurface. 

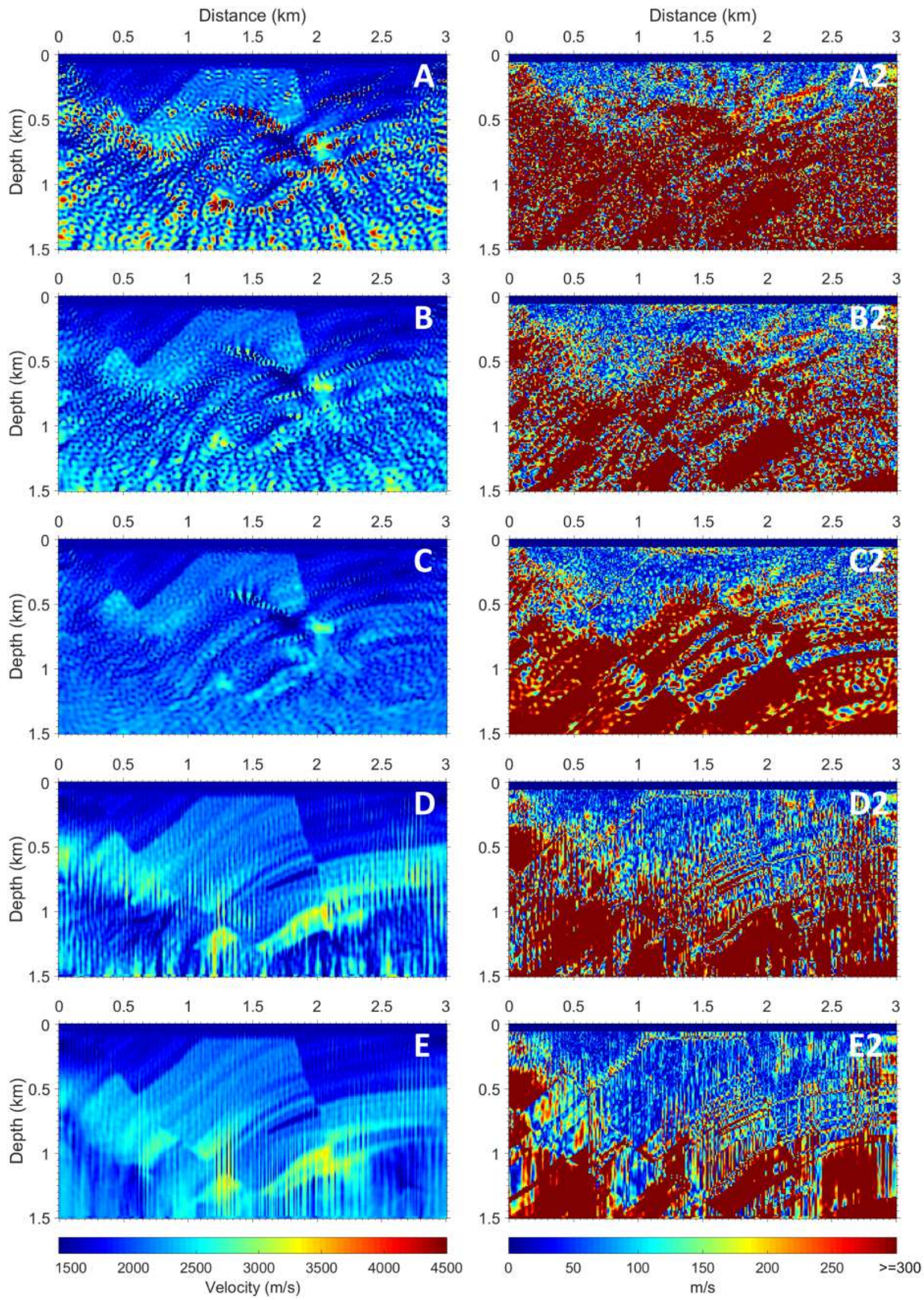

Figure 2 - $P$-velocity models obtained from the traditional objective function ( $l_{2}$-norm of the residuals) (A), traditional Bayesian formulation (B), Traditional Bayesian formulation with our proposal (C), Bayesian formulation with a priori knowledge regarding statistical correlations between the P-velocities (D) and Bayesian formulation with a priori knowledge regarding statistical correlations between the $P$-velocities and with our proposal $(E)$ and corresponding differences (in absolute value) with the true model (in the right column).

Sixteenth International Congress of the Brazilian Geophysical Society 


\section{ACKNOWLEDGMENTS}

The authors gratefully acknowledge support from Shell Brasil through the "New Methods for Full Waveform Inversion" project at Federal University of Rio Grande do Norte (UFRN) and the strategic importance of the support given by ANP through the R\&D levy regulation. The authors also gratefully acknowledge support from Brazilian National Council for Scientific and Technological Development (CNPq).

\section{References}

Asnaashari, A., Brossier, R., Garambois, S., Audebert, F., Thore, P., and Virieux, J., 2013, Regularized seismic full waveform inversion with prior model information: Geophysics, 78, no. 2, R25-R36.

Bui-Thanh, T., Ghattas, O., Martin, J., and Stadler, G., 2013, A computational framework for infinitedimensional bayesian inverse problems. part i: the linearized case, with application to global seismic inversion: SIAM Journal on Scientific Computing, 35, no. 6, A2494-A2523.

Carvalho, P., Corso, G., and Lucena, L., 2018, Fullwaveform inversion with long-range correlations: Rio Oil Gas Expo and Conference 2018.

Komatitsch, D., and Martin, R., 2007, An unsplit convolutional perfectly matched layer improved at grazing incidence for the seismic wave equation: Geophysics, 72, no. 5, 155 â167.

Lailly, P., 1983, The seismic inversion problem as a sequence of before stack migrations: Conference on Inverse Scattering, Theory and Application, 206-220.

Li, Y. E., and Demanet, L., 2016, Full-waveform inversion with extrapolated low-frequency data: Geophysics, 81, no. 6, R339-R348.

Mandelbrot, B., and van Ness, J. W., 1968, Fractional brownian motions, fractional noises and applications: Society for Industrial and Applied Mathematics, 10, no. 4, 422-437.

Martin, G. S., Wiley, R., and Marfurt, K. J., 2006, Marmousi2: An elastic up-grade for marmousi: The Leading Edge, 25, 156-166.

Mosegaard, K., and Tarantola, A., 2002, Probabilistic approach to inverse problems in Lee, W. H., Kanamori, H., Jennings, P. C., and Kisslinger, C., Eds., International Handbook of Earthquake and Engineering Seismology, Part A:: Academic Press, 237 - 265.

Pasalic, D., and McGarry, R., 2010, Convolutional perfectly matched layer for isotropic and anisotropic acoustic wave equations:.

Sahimi, M., and Tajer, S. E., 2005, Self-affine fractal distributions of the bulk density, elastic moduli, and seismic wave velocities of rock: Physical Review E.

Tarantola, A., 1984, Inversion of seismic reflection data in the acoustic approximation: Geophysics, 49 , no. $8,(8)$, 1259-1266.
Tarantola, A., 1987, Inverse problem theory: Methods for data fitting and model parameter estimation: Elsevier Science Publication Company, Inc.

Tejero, C. E., Dagnino, D., Sallares̀, V., and Ranero, C. R., 2015, Comparative study of objective functions to overcome noise and bandwidth limitations in full waveform inversion: Geophysical Journal International, 203, no. 1, 632-645.

Virieux, J., and Operto, S., 2009, An overview of full-waveform inversion in exploration geophysics: Geophysics, 74, no. 6, WCC1-WCC26.

Virieux, J., Asnaashari, A., Brossier, R., Métivier, L., Ribodetti, A., and Zhou, W., 2017, An introduction to full waveform inversion: Encyclopedia of Exploration Geophysics, pages R1-1âR1-40. 